\title{
I SPEAK, THEREFORE I AM
}

The importance of good usage of language in psychiatrists has been sorely underemphasised. We would expect surgeons to be good with their hands, and internists to be thorough and systematic, working through problems analytically. Similarly, psychiatrists, by the very nature of their work, ought to be great communicators, able to build rapport with patients easily and tailor their conversations according to the different profiles of the people they meet. Lamentably, the importance of language is often understated in medical school, and even during specialist training in psychiatry. Psychiatrists are trained in-depth to elicit responses and interpret psychopathology through various interviewing techniques and skills. However, this process can very often be challenging if one does not have a good foundation in the native language used, or if the psychiatrist is not fluent in the use of that particular medium of instruction. I would thus like to stress the importance of a good grounding in language - in particular the spoken language - in a psychiatrist's armamentarium of everyday skills. Language skills is by far the most important tool for a psychiatrist, for without effective use of language, all the knowledge of psychopathology and interview skills cannot be put into action. Language skills in a psychiatrist is thus akin to the hands of a surgeon - skilful use of language helps a psychiatrist to dissect and analyse the mind of the patient he or she is treating.

Psychiatrists work by getting detailed histories from patients, corroborating these histories with accounts given by the patients' relatives and friends, performing mental state assessments, ordering investigations and scans, coming up with a formulation and finally using this formulation to generate a treatment plan. In psychiatry, the formulation is highly important as it is here that psychiatrists recognise the contributions of biological, psychological and social factors in the aetiology of most psychiatric illnesses. This is different from the way medicine or surgery is practised. Formulation is not stressed as much in the latter fields, as the diagnosis is enough to point the doctor towards a treatment plan. The reaching of a diagnosis in medicine or surgery is determined by good focused history, good clinical examination, and very importantly in this day and age, investigations by scans and other diagnostic tests (for example, biopsy). Conversely in psychiatry, diagnoses and formulations are largely reached based on history gathered. The amount of physical examination and investigations that need to be ordered are generally minimal. In order to obtain a meaningful and in-depth history, good verbal usage of language as well as good interview skills are necessarily involved, with the latter being highly dependent on the former. Hence, the medical education of a good psychiatrist should not merely entail the inculcation of good interview techniques and skills, but should also include further training in the use of language. Such training could include vocabulary expansion, education on the use of a variety of expressions and body language, and even the learning of the nuances of certain words and colloquial terms used in the local context. Even the way certain inflexions are emphasised could alter the meaning of a sentence. This can be skilfully applied by psychiatrists if proper training were conducted.

One may think that I am quibbling by asking for psychiatrists to be adept in the use of language. One may also argue that the criteria for placement in medical school have already selected people with an adequate command of the language that would enable them to go through the rigours of studying medical textbooks. However, after having practised in the field of psychiatry for three years and as a psychiatry registrar for about a year, I frequently observe psychiatrists who differ in their opinions on what the patient is suffering from. This observation has also been noticed by many of my colleagues. While some of these differences may be due to psychiatrists seeing patients at different time points (hence allowing for the possibility of the evolution of the illness), sometimes the differences in opinions cannot be explained by time intervals, especially when the patient is seen almost immediately after by another psychiatrist.

What, then, causes this difference in diagnoses between two psychiatrists who are both well trained in psychopathology and Diagnostic and Statistical Manual of Mental Disorders-IV diagnoses? Perhaps an important contributory factor is the application of the spoken language. Not much research has been done on the relationship between the language skills and effectiveness of psychiatrists, as the logic in this correlation is perhaps apparent. However, in this day and age of globalisation where doctors and psychiatrists practise in various parts of the world away from their homeland, this issue has become even more pertinent, particularly in the field of psychiatry where there is a heavy reliance on language. To illustrate, the words "depressed" and "obsessed" have become quite loosely used such that their usage sometimes detracts from their original psychiatric definitions when used by patients. Thus, it is up to the psychiatrist to tease out the exact meaning of such terms when used by patients, so as to fully elucidate the nature of the patient's complaints. To do so effectively would entail the psychiatrist's skilful use of language, rather than accepting at face value what the patient says. 
Psychiatry has seen much advancement in recent years, with the increasing use of radiological investigations and biochemical markers to assist in the diagnosis of certain conditions such as $\mathrm{N}$-methyl D-aspartate encephalitis, frontotemporal dementia, etc. However, psychiatric conditions frequently require no more than the effective use of language and communication skills to diagnose and formulate, and this emphasis should be stressed in undergraduate medical education as well as subsequent specialist training. Psychiatrists are not just doctors, but also effective communicators and speakers. This is our niche and we need to do ourselves proud.

Yours sincerely,

Lambert Low Tchern Kuang, MBBS, MRCPsych

National Addictions Management Service, Institute of Mental Health, Singapore. Lambert_Tk_LOW@imh.com.sg 